\title{
Switching Frames for Constant Bit Rate Video Streams
}

\author{
M. Altaf \\ School of Computer Science and \\ Electronic Engineering \\ University of Essex, UK \\ +44 1206-874448 \\ maltaf@essex.ac.uk
}

\author{
E. Khan \\ Dept. of Electronics Engineering \\ Aligarh Muslim University \\ Aligarh, India \\ ekhan@lycos.com
}

\author{
M. Ghanbari \\ School of Computer Science and \\ Electronic Engineering \\ University of Essex, UK \\ +44 1206-872826 \\ ghan@essex.ac.uk
}

\begin{abstract}
To map the data rate to the available channel bandwidth, one method is to switch between video streams of different qualities using either intra (I-) frames or the switching predictive (SP-) frames of H.264. Due to its better coding efficiency SP-frames are preferred over I-frames, but these frames are generally exercised with variable bit rate (VBR) coded videos, therefore limiting their usage. For constant bit rate (CBR) coded videos, in which the quantization parameters (QP) are decided on the fly for every macro block, the design of SP-frames becomes difficult due to the multiple (twice) quantizations used in SP-frames. In this paper we propose to design SP-frames for CBR coded videos, selecting the QPs in such a way that the varying QP nature of CBR coding is preserved. The proposed algorithm is compared with the already implemented SP-frame concept for VBR video coding, showing that both the concepts have similar behavior.
\end{abstract}

\section{Categories and Subject Descriptors}

C.2.1 [Computer-Communication Networks]: Network communications---Wireless communication; H.3.4 [Information Storage and Retrieval]: Performance evaluation (efficiency and effectiveness); H.5.1 [Multimedia Information Systems]: Video; I.4.1 [Digitization and Image Capture]: Quantization

\section{General Terms}

Measurement, Performance. Multimedia

\section{Keywords}

Constant bit rate, H.264/AVC, Switching frames, Variable bit rate, Video Streaming

\section{INTRODUCTION}

One of the most important goals for which a video streaming system is designed, is the continuous reception of video stream without any interruption [1]. Due to the varying bandwidth nature of today's mobile networks, one of the key problems in the video streaming is to match the data rate of the transmitted video

Permission to make digital or hard copies of all or part of this work for personal or classroom use is granted without fee provided that copies are not made or distributed for profit or commercial advantage and that copies bear this notice and the full citation on the first page. To copy otherwise, or republish, to post on servers or to redistribute to lists, requires prior specific permission and/or a fee.

MobiMedia'09, September 7-9, 2009, London, UK.

Copyright 2009 ICST 978-963-9799-62-2/00/0004 _... \$5.00. according to the varying network conditions [2].

Various techniques are proposed in the literatures to sort out this problem [3]-[6]. Among these, dynamic switching among different independently encoded bit streams of the same video, having different quality and hence different bit rates [1][7] is widely used. This has been successfully implemented in applications like "SureStream" of real networks and "Intelligent streaming" from windows media using periodically inserted Iframes for switching between the streams [8]-[10].

In H.264/AVC, SP-frames have been defined [11]-[13] for the drift free switching among multiple bit streams instead of I-frames due to their better coding efficiency. H.264/AVC specifies two types of switching frames, namely the primary SP (PSP-) frames and the secondary SP (SSP-) frames. They use motion compensated predictive coding and thus have better compression efficiency than the I-frames [11]-[12], albeit at relatively lower quality. As long as switching is not desired PSP-frames are transmitted instead of P-frames at the preselected positions. If switching becomes necessary, an SSP-frame, which is a mismatch free version of the PSP-frame, is transmitted at the switching instance, replacing the PSP-frame at that position.

As the SP-frames are defined for VBR coded videos only, limiting their benefits to only VBR coded videos. Therefore it is an indisputable requirement to design SP-frames for the CBR coded video to increase its area of application and hence to improve quality of service (QoS) by efficiently matching the data rate to the available bandwidth.

As the SP-frames use two user defined quantization parameters [15]-[17], but in almost all the codecs (including H.264/AVC), the generation of CBR bitstreams requires the use of one QP selected according to the rate distortion optimization [14]. Thus the problem of CBR bitstream switching using SP-frames is that how to select the QP pair for these frames without sacrificing the varying $\mathrm{QP}$ nature of the CBR encoder.

This paper addresses this issue and proposes a solution based on the characteristics of SP-frames, selecting QP pair for these fames in such a way that this basic nature of the CBR video is preserved. Simulation results show that our proposed concept of switching frames of the CBR has similar behavior to that of the VBR and can be used for drift free switching between CBR video streams of different bit rates. 


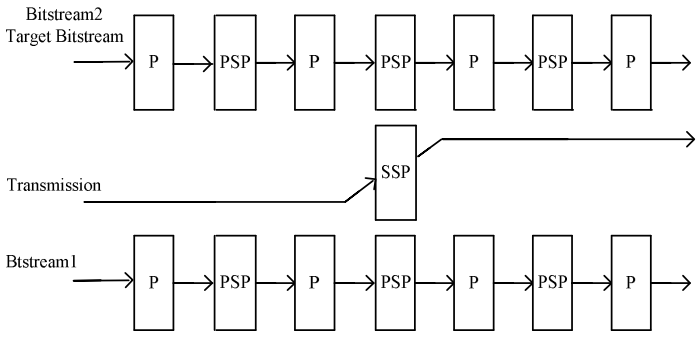

Figure 1. Bitstream Switching using SP-frames.

The remaining parts of the paper are organized as follows: the switching frames and the effects of quantization parameters are investigated in section II. Section III describes switching frames for CBR video. Section IV includes simulation results and finally concluding remarks are given in section $\mathrm{V}$.

\section{ANALYSIS OF VBR SP-FRAMES}

SP-frames have been defined in H.264/AVC for drift free switching between multiple copies of the same video coded with different qualities [11]-[12]. The whole switching concept is carried out in two steps using two switching frames, namely PSPframes and SSP-frames as shown in Fig. 1. PSP-frames are inserted at frame location where switching is desired, If switching is required, the SSP-frame is transmitted instead of PSP-frame at that frame position [11]-[12]. These are motion compensated predictive frames and thus have better compression efficiency than the I-frames. The PSP-frames are coded similar to the standard P-frames while the SSP-frames have special encoding which leads to the same reconstructed frame as the primary SPframe, even if a different reference frame is used [18].

A PSP-frame is quantized two times, first coding prediction error (QSP) and second predicted blocks (QSSP) before forwarding to the reference frame buffer. This second quantization parameter enables drift free reconstruction of PSP-frame in the form of SSPframe from different reference frames. As double quantization parameters increases quantization errors therefore the quality of these frames is usually lower than that of the P-frames. To reduce the quantization errors the values of QSP and QSSP are taken lower than the QP [12][17] giving an equation similar to (1) below

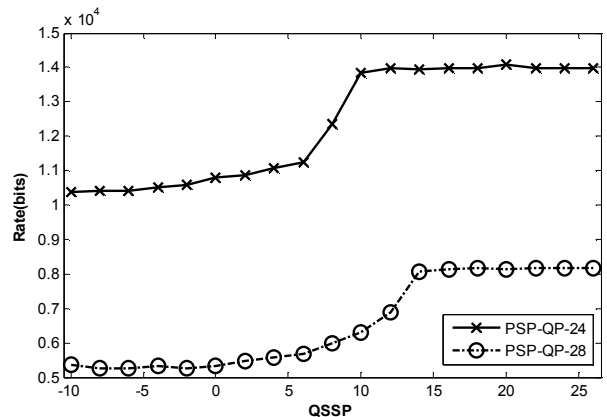

Figure 2. Bit rate of PSP-frames

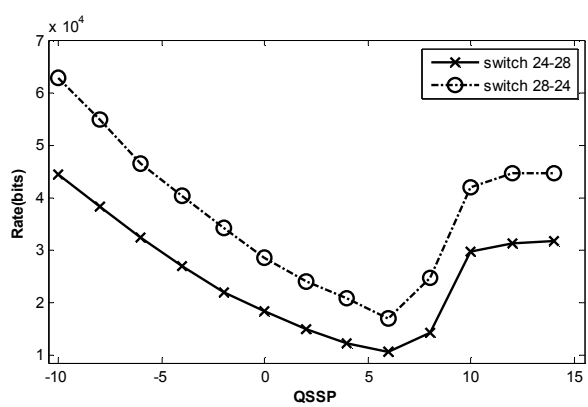

Figure 3. Bit rate of SSP-frames

$Q S P=Q P-i$
$Q S S P=Q P-j$

Where $i$ and $j$ are two integers ranging from 0 to QP.

QSP is the main quantization parameter of the PSP-frame, playing the same role as QP in other frames. Therefore its behavior is very straight forward and is not discussed here. The QSSP on the other hand is a new concept which affects the reference PSPframe before being stored in the reference frame buffer and hence is discussed in detail in the following sub-section.

\subsection{Effects of QSSP}

To study the effects of the QSSP on the switching frames, a wide range of quantization parameters are tested using different video sequences. Here we consider a QCIF video sequence, namely "Foreman", coded at $10 \mathrm{fps}$, with GOP size of 10 having I-frame at the GOP boundaries or a PSP-frame after every $10^{\text {th }}$ frame. The effects of QSSP on PSP-frame is shown in Fig. 2 for the "Foreman" sequence, keeping QSP constant and equal to QP, which in this particular test is set to 24 and 28.

$\mathrm{X}$-axis shows the offset of the QSSP from the QP of the rest of the frames (I and P) of the sequence. The bit rate is averaged over a GOP of 10 frames including that of a PSP-frame at the GOP boundary. It can be seen from Fig. 2 that as QSSP increases, the bit rate of the PSP-frame increases and the reason behind this is that as the QSSP increases so the distortion of the reference frame, and at higher distortion it becomes difficult to predict the current PSP-frame from such a distorted reference frame. Therefore the encoder increases the number of intra-coded macro blocks in the PSP-frame avoiding motion compensation, which in response increases the bit rate of the PSP-frames as shown in Fig. 2. Therefore keeping the QSSP less than QSP or very close to it can help in keeping the bit rate low.

In the case of switching between streams of different bit rates, the SSP-frame having QSSP as the main quantization parameter is used. The PSNR of the SSP-frame is equal to that of PSP-frame as an exact reconstruction is required. The bit rate of the SSPframe not only depends on the value of the QSSP but also on the direction of switching i.e. switching from low bit rate to high bit rate (up switching) or switching from high bit rate to low bit rate (down switching) as shown in Fig. 3 for "Foreman" sequence for both up switching (QP 28 to QP 24) and down switching (QP 24 to QP 28). 
Here the bit rate of only SSP-frame (instead of taking the average over a GOP) is plotted, as the bit rate of the frames before and after SSP-frame remains the same as was in the sequences with PSP-frames. The bit rate of the SSP-frame in case of up switching is higher than the SSP-frame of down switching. The reason is that in up switching the reference frame is of lower quality and the frame to be coded (SSP-frame) is of higher quality resulting in higher error signal and hence higher bit rate. Similarly the down switching results in lower error signal due to higher quality reference frame and hence decreasing the bit rate.

\section{DESIGN OF CBR SP-FRAMES}

In CBR coded video the quantization parameters are calculated for each and every macro block according to the rate distortion optimization. Therefore the main problem in designing switching frames for the CBR video is the selection of the QSP and QSSP such that the varying nature of quantization parameter in CBR coding is not violated.

As the switching frames are motion compensated predictive frames and the only difference between the PSP-frame and the Pframe is the extra quantization of PSP-frame before feeding it to the reference frame buffer. Therefore the value of the main quantization parameter QSP is decided by the encoder without introducing any changes in the encoder RDO operation, while the QSSP is selected as a function of the QSP according to Eqn. (2).

$$
\left.\begin{array}{l}
Q S P=Q P \\
Q S S P=Q S P-j
\end{array}\right\}
$$

Where QP is the quantization parameter of P-frame and $j$ is an integer ranging from 0 to QSP. As discussed in section 2.1, QSSP helps in reducing the bit rate of PSP-frame by increasing the quality of the reference frame. The values of QSSP are set to less than QSP so that the bit rate is kept at minimum, reducing burden on the overall bit budget of the sequence. The selection of QSP equal to QP will not violate the nature of CBR coding as the main quantization parameter QSP is selected by the encoder according to its RDO criterion.

R-D comparison of the CBR and VBR coded video are shown in figures 4 ' $a$ ' and ' $b$ ' for "Foreman" sequence. Average PSNR vs. bit rate curves for one GOP are drawn for the QSSP values "QSP1 " and "QSP-2" along with that of I and P-frames, coding these sequences with I-frames at GOP instead of PSP-frames and with

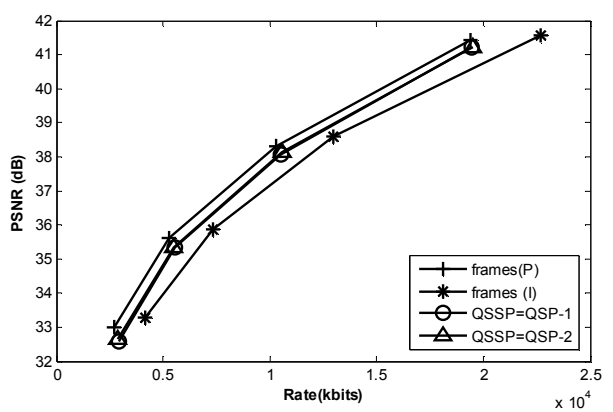

(a)

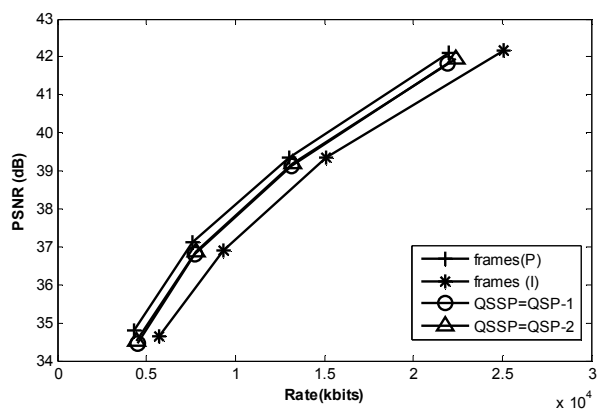

(b)

Figure 4. R-D curves for PSP-frame "Foreman" (a) VBR (b) CBR

P-frames at all locations with first frame as intra coded respectively. For comparison the bit rate and initial QP of the CBR video is kept equal to that of the VBR video. In both the sequences it can be seen that the behavior of the CBR and VBR PSP-frames are similar supporting our method of the CBR switching frames.

\section{SIMULATION RESULTS}

Extensive simulations were carried out using H.264/AVC (JM 10.2 as the SP-frames are not supported in the versions after JM12) encoder with necessary changes. The results are compared for both VBR and CBR SP-frames. For PSP-frames the average bit rate and PSNR for the GOP are compared, while in the case of secondary switching frames the bit rate and PSNR of the

Table 1 Comparison of VBR and CBR switching frames

\begin{tabular}{|c|c|c|c|c|c|c|c|c|c|c|}
\hline \multirow{3}{*}{\multicolumn{2}{|c|}{ Sequence }} & \multicolumn{3}{|c|}{$\begin{array}{c}\text { QSP }=\mathbf{Q P} \\
\mathbf{Q S S P}=\mathbf{Q S P}\end{array}$} & \multicolumn{3}{|c|}{$\begin{array}{c}\mathrm{QSP}=\mathrm{QP} \\
\mathrm{QSSP}=\mathrm{QSP}-3\end{array}$} & \multicolumn{3}{|c|}{$\begin{array}{c}\mathrm{QSP}=\mathrm{QP} \\
\mathrm{QSSP}=\mathrm{QSP}-6\end{array}$} \\
\hline & & \multicolumn{2}{|c|}{ PSP } & \multirow{2}{*}{$\begin{array}{c}\text { SSP } \\
\text { (bits) }\end{array}$} & \multicolumn{2}{|c|}{ PSP } & \multirow{2}{*}{$\begin{array}{c}\text { SSP } \\
\text { (bits) }\end{array}$} & \multicolumn{2}{|c|}{ PSP } & \multirow{2}{*}{$\begin{array}{c}\text { SSP } \\
\text { (bits) }\end{array}$} \\
\hline & & (bits) & (dB) & & (bits) & (dB) & & (bits) & $(\mathrm{dB})$ & \\
\hline Foreman (VBR) & & 5572 & 35.23 & 19272 & 5352 & 35.36 & 26712 & 5260 & 35.46 & 34752 \\
\hline Foreman (CBR) & & 7825 & 36.79 & 24568 & 7666.4 & 36.91 & 32520 & 7652 & 36.981 & 42424 \\
\hline Akyio (VBR) & & 2887 & 40.56 & 21804 & 2771 & 40.73 & 27912 & 2719 & 40.95 & 35056 \\
\hline Akyio (CBR) & Q 11 (3) & 2664 & 39.75 & 1639 & 2661 & 39.82 & 18664 & 2484 & 40.22 & 26144 \\
\hline
\end{tabular}


individual SSP fames are compared for both up switching and down switching and is summarized in Table 1.

In Table 1, the first row shows the equations of the QSP and QSSP, while first column shows the sequence names and QPs in parenthesis for SSP-frames switching from first value of QP to second value. The second value of the QP in parenthesis is also used as QP of the rest of the frames of the stream including PSPframes. The quantization parameter for the CBR video are the initial quantization parameters that keep on changing according to the RDO method, here they are written in order to make comparison easier with the VBR video. The values of the QSSP are taken as a function of the QSP, decreased by 3 from left to right.

It can be seen that as we go from left to right in Table 1, the bit rate for PSP-frames decreases and the PSNR increases in both types of coding modes showing similar behavior of the switching frame in both the cases. This decrease in bit rate and increase in the PSNR from left to right is the effect of the QSSP, as the QSP remains constant. Similar behavior can be observed for the SSPframes; i.e. from left to right the changes in bit rate (increase or decrease) is similar in both the coding modes. The bit rate of the SSP-frame increases from left to right due to the decrease in the value of QSSP. PSNR values for SSP-frames are not shown as they are similar to the PSP-frames. The values of the bit rate and PSNR are not exactly equal in VBR and CBR coding, and the reason is due to varying QP of the CBR coding but it clearly shows that the behavior of switching frames in both the coding modes is similar, supporting our concept of CBR switching.

\section{CONCLUSION}

Rate distortion characteristics of the switching frames were analyzed in detail, with the effects of QSP and QSSP on the bit rate and PSNR of SP-frames. In this paper we extended the switching frames concept to CBR video and compared the rate distortion characteristics of this new approach with existing switching frames concept of the VBR video. The main problem was the selection of QSP and the QSSP in the CBR scenario where the quantization parameters keep changing. In the proposed solution, the encoder decides the value of the QSP based on R-D criterion, and QSSP which is used for reference PSP-frame is selected as a function of the current QSP. In this way the varying QP nature of the CBR coding is maintained.

Simulations showed that the behavior of these CBR SP-frames is found to be similar to that of the VBR SP-frames. The CBR concept of SP-frames can easily replace the intra frames in the widely implemented CBR video streaming, enabling switching between CBR video streams of the same sequence coded with different bit rates, and hence increasing resource utilization.

\section{REFERENCES}

[1] Apostolopoulos, J. G., Tan, W. T. and Wee, S. J. 2002 Video Streaming: Concepts, Algorithms, and Systems. Technical Report. HP Laboratories Palo Alto, HPL-2002260.

[2] J. Cai, He, Z., and Chen, C. W. Rate-reduction transcoding design for video streaming applications. In proceedings of IEEE Packet Video Workshops, (PV' 2002), (Pittsburg PA, USA, 2002).
[3] Foster, I. and Roy, A. A Quality of Service architecture that combines resource reservation and application adaptation. In proceedings of Eight International Workshop on Quality of Service (IWQoS 2000), (Pittsburg PA, USA, 2000) 181-188.

[4] Feamster, N., Bansal, D. and Balakrishnan, H. On the interaction between layered quality adaptation and congestion control for streaming video. In Proceedings of 11th International Packet Video Workshop (PV2001), (Kyongiu, Korea, 2001).

[5] Wien, M., Schwarz, H. and T. Oelbaum, Performance Analysis of SVC, IEEE Transactions on Circuits and Systems for Video Technology, 17 (9) (Sep 2007) 11941203.

[6] Schwarz, H. and Wien, M. The Scalable Video Coding Extension of H.264/AVC Standard. IEEE Signal Processing Magazine, (March 2008) 135-141.

[7] Xie, B. and Zeng, W. Fast Bitstream Switching Algorithms for Real-Time Adaptive Video Multicasting. IEEE Transactions on Multimedia, 9 (1) (Jan 2007) 169-175.

[8] SureStream Technology http://service.real.com/help/library/guides/productiong27/ht mfiles/bandwdth.htm

[9] Intelligent Streaming http://www.microsoft.com/windows/windowsmedia/howto/ar ticles/intstreaming.aspx

[10] Girod, B. Video Over Networks http://www.stanford.edu/class/ee398b/handouts/lectures/08VideoOverNetworks.pdf

[11] Karczewicz, M. and Kurceren, R. The SP- and SI-Frames Design for H.264/AVC. IEEE Transactions on Circuits and Systems for Video Technology, 13 (7) (Jul 2003) 637-644.

[12] Stockhammer, T., Liebel, G., and Walter, M. Optimized H.264/AVC-Based Bit Stream Switching for Mobile Video Streaming. EURASIP Journal on Applied Signal Processing,(2006) 1-19.

[13] Setton, E. and Girod, B. Rate-Distortion Analysis and Streaming of SP and SI Frames. IEEE Transactions on Circuits and Systems for Video Technology, 16 (6) (June 2006) 733-743.

[14] Ma, S., Gao, W. Rate-Distortion analysis for H.264/AVC video coding and its application to rate control. IEEE Transactions on Circuits and Systems for Video Technology, 15(12) (Dec 2005)1533-1544.

[15] Yang, X., Tan, Y., Ling, N. Rate Control for H.264 with two step quantization parameter determination but single-pass encoding. EURASIP Journal on applied signal processing, (2006) 1-13

[16] Wiegend, T., Schwarz, H., Joch, A., and Kossentini, F. Rateconstrained coder control and comparison of video coding standards. IEEE Transactions on Circuits and Systems for Video Technology, 13 (7) (Jul 2003) 688-703.

[17] Setton, E., Ramanathan, P. and Girod, B. Rate-distortion analysis of SP and SI frames. In proceedings of Visual Communication Image Processing (VCIP), (San Jose, CA, 2006).

[18] Walter, M. 2004 Advanced Bit stream switching for Wireless for Wireless Video Streaming. Diploma Thesis, Institute for communication Engineering, Munich University of Technology. 